\title{
MAGNETISM IN RARE-EARTH-TRANSITION METAL SYSTEMS. MAGNETIZATION REVERSAL AND ULTRA-HIGH SUSCEPTIBILITY IN SANDWICHED THIN FILMS BASED ON RARE-EARTH AND COBALT ALLOYS
}

\author{
L.T. Baczewski ${ }^{a}$, D. Givord ${ }^{b}$, J.M. Alameda ${ }^{c}$, B. Dieny ${ }^{b}$, \\ J.P. Nozieres ${ }^{b}$, J.P. REBoulllat ${ }^{b}$ and J.J. PrejeaN ${ }^{d}$ \\ ${ }^{a}$ Institute of Physics, Polish Academy of Sciences \\ Al. Lotników 32/46, 02-668 Warszawa, Poland \\ b Laboratoire Louis Néel, CNRS, 166X, 38042 Grenoble, France \\ c Departamento de Physica, Universidad de Oviedo, Oviedo, Spain \\ ${ }^{d}$ CRTBT, CNRS, 166X, 38042 Grenoble, France
}

This paper is composed of two parts: theoretical and experimental. The theoretical part is divided into several sections. The main characteristic properties of $3 d$ (transition metals) and $4 f$ (rare-earth metals) magnetism are explained in the second section. The properties of rare-earth-transition metal compounds are discussed in the third section, particularly with respect to applications. The fourth section is devoted to the rapidly growing field of magnetic thin films. Specific properties of ultra-thin films and multilayers are summarized. In the experimental part the original magnetic properties of sandwich films, consisting of a soft amorphous Co-Zr layer placed between two Sm-Co layers with different coercivities, are reported.

PACS numbers: 75.30.-m, 75.30.Gw, 75.70.-i, 75.70.Fr

\section{Introduction}

A rapid evolution characterizes the magnetic materials in the domain of permanent magnets and in the one of magnetic recording. Further advances are already in progress, especially those related to the thin films and multilayers technology. In this context, the understanding of basic magnetic properties of such materials is necessary.

The main characteristic properties of $3 d$ (transition metals) and $4 \int$ (rare-earth metals) magnetism are explained in Sec. 2. The properties of rare earth (R)-transition metal (M) compounds are discussed in Sec. 3, particularly with rcspect to applications. Section 4 is devoted to the rapidly growing field of magnetic 
thin films. Specific properties of ultra-thin films and multilayers are summarized. In the last, fifth section we report some original experimental results of sandwich films composed of a soft Co-Zr layer placed between two Sm-Co layers with different coercivities.

\section{2. $3 d$ and $4 f$ magnetism in metallic systems}

The definitions of the spin and orbital magnetic moments and the origin of magnetism in the two principal series, $3 d$ and $4 f$, are recalled. The essential properties of $3 d$ metals (large exchange interactions) and those of $4 f$ metals (large magnetocrystalline anisotropy) are described.

\subsection{Magnetic moment}

An electron moving on a simple circular orbital creates a magnetic field which, according to the Biot and Savart law, is

$$
B=\frac{\mu_{0}}{4 \pi} \frac{e v \times r}{r^{3}} .
$$

From the basic formulae of magnetostatics, the associated orbital magnetic moment is

$$
\begin{aligned}
m_{L} & =\frac{e v \times r}{4 \pi r^{3}} \times \frac{4}{3} \pi r^{3} \\
& =\frac{e v \times r}{3}
\end{aligned}
$$

with $e=1.6 \times 10^{-19} \mathrm{C}, v=10^{6} \mathrm{~m} / \mathrm{s}, r=10^{-10} \mathrm{~m}, m_{L}$ is evaluated to be $0.5 \times 10^{-23} \mathrm{~A} \cdot \mathrm{m}^{2}$. Actually, the basic unit of magnetic moment is the Bohr magneton, $\mu_{\mathrm{B}}$, equal to $0.92 \times 10^{-23} \mathrm{~A} \cdot \mathrm{m}^{2}$.

In addition to the orbital magnetic moment, a spin magnetic moment $m_{\mathbf{s}}$ of $1 \mu_{\mathrm{B}}$ is attached to each electron. The classical analogy for the spin moment is that of the rotation of the electron about itself. The spin moment aligns itself along the field created at its position by the orbital moment, this is the spin-orbit coupling.

When the electrons are localized, an atomic magnetic moment may be defined. It results from the combination of individual spin and orbital moments, according to the Hund rules. It is fairly obvious that electrons tend to occupy different orbitals in order to reduce their electrostatic repulsion. Moreover, $\sum m_{\mathbf{s}}$ tends to be maximum, i.e. the spins of electrons on different orbitals are parallel. In such instance, the electrostatic repulsion is further decreased, since the probability for one electron to hop from one orbital to another is strictly equal to zero according to the Pauli exclusion principle. The above interactions, of electrostatic origin, thus favour the formation of a moment at the atomic scale.

On a complete shell, the individual contributions of the different electrons cancel out. Magnetism is a property of uncompleted shells. There are two main series of magnetic elements. From transition metals $\mathrm{Cr}$ to $\mathrm{Ni}$, the magnetic moment is due to electrons on the $3 d$ shell. From rare-earth metals $\mathrm{Ce}$ to $\mathrm{Yb}$, the magnetic moment is due to electrons on the $4 f$ shell. 


\subsection{Magnetism in $3 d$ metals: large exchange interactions}

Strong coupling between magnetic moments is observed in general in $3 d$ metals. In particular, ferromagnetism is stable in $\mathrm{Fe}, \mathrm{Co}, \mathrm{Ni}$ up to temperatures much higher than room temperature. In fact, the $3 d$ electrons occupy an outer shell and in metals they participate in the band structure. Their itinerant character is at the origin of the coupling between atomic moments. As electrons hop from site to site, the direction of their spin is preserved on a characteristic length of the order of the Fermi wavelength $\lambda_{\mathrm{F}}$. In Co and $\mathrm{Ni}$, the $3 d$ band is nearly full and $\lambda$ is much larger than interatomic distances. Ferromagnetic coupling of the moments occurs which ensures at any time parallelism of the spin moments of all electrons at all atomic sites. In $\mathrm{Mn}$, the $3 d$ band is about half-filled and $\lambda_{\mathrm{F}}$ is of the order of interatomic distances. Antiferromagnetic coupling in this instance is observed. $\mathrm{Fe}$ is in an intermediate situation, both ferromagnetic $(\alpha-\mathrm{Fe})$ and antiferromagnetic $(\gamma-\mathrm{Fe})$ coupling may occur.

\subsection{Magnetism in $4 f$ metals: large magnetic anisotropy}

In rare-earth metals, the temperatures of magnetic ordering are in general lower than room temperature. This shows that the magnetic interactions between spin moments are much weaker than in $3 d$ metals. In fact, the $4 f$ electrons occupying an inner shell are strongly localized and no direct interaction may occur. The exchange interactions between $4 f$ electrons are indirect, mediated by $5 d, 6 s$ electrons.

A specific property of these metals is the occurrence of a large magnetocrystalline anisotropy in the ordered magnetic state. The spin and orbital moments of rare-earth atoms may be large and are maintained parallel to each other by strong spin-orbit coupling ( $J=L-S$ for light R elements $\mathrm{Pr}, \mathrm{Nd}, \mathrm{Sm} ; J=L+S$ for heavy $R$ elements $\mathrm{Tb}, \mathrm{Dy}, \ldots)$. The magnetocrystalline anisotropy then results from the interplay between exchange and crystalline electric field (CEF) interactions acting on the $4 f$ electrons. Let us assume for simplicity that the exchange interactions are larger than CEF interactions. The ground state of the $4 f$ electrons is that of maximum moment. In $\mathrm{Sm}, \mathrm{Er}, \mathrm{Tm}, \mathrm{Yb}$, the $4 f$ electron density is prolate, i.e. it is elongated along the direction of the magnetic moment. In $\mathrm{Pr}, \mathrm{Nd}$, $\mathrm{Tb}, \mathrm{Dy}, \mathrm{Ho}$, the $4 f$ electron density is oblate. In a given structure, the orientation of the $4 f$ electron density is defined by the electrostatic potential created by the charges of the environment. In the hexagonal structure of the $\mathrm{R}$ metals, it tends to be elongated along $c$. This leads to the easy direction magnetization being along $c$ for $\mathrm{Sm}, \mathrm{Er}, \mathrm{Tm}, \ldots$ and perpendicular to it for $\mathrm{Nd}, \mathrm{Pr}, \ldots$ Typical value of the anisotropy energy at $4.2 \mathrm{~K}$ is $10^{8} \mathrm{~J} / \mathrm{m}^{3}$. However, as temperature increases, the anisotropy strongly decreases and finally vanishes at the ordering temperature, i.e. below room temperature.

\section{Magnetic properties of $\mathbf{R}-\mathbf{M}$ compounds}

The value of the $3 d$ magnetic moment and $3 d$ exchange interactions in $\mathrm{R}-\mathrm{M}$ compounds are discussed. An antiparallel coupling between $R$ and $M$ spins is 
shown to occur and the strength of the $\mathrm{R}-\mathrm{M}$ interactions is evaluated. The magnetic anisotropy in compounds is discussed. It is shown, in particular, that large exchange interactions and magnetocrystalline anisotropy may be obtained in $\mathrm{R}-\mathrm{M}$ rich compounds crystallizing in a structure of uniaxial symmetry.

\subsection{Decrease in the $3 d$ moment with the percentage of $R$ atoms alloyed}

In $\mathrm{R}-\mathrm{M}$ intermetallic compounds, when $\mathrm{R}$ is a nonmagnetic element, a decrease in the $3 d$ magnetic moment is observed as the percentage of $R$ elements is increased [1,2]. For $Y$ concentration of up to $y=0.2$ a progressive decrease in the $3 d$ moment, in both Fe and Co compounds, occurs. For $y>0.2$, the Co moment decreases very rapidly.

The above behaviour is usually discussed in terms of electron transfer of $5 d$ electrons into the $3 d$ band, and $5 d-3 d$ hybridization effects. More generally, Williams et al. [3] have discussed the variation of the magnetization in several series of alloys using Friedel's interpretation [4] of the Slater-Pauling curve for transition metal alloys. In this approach, the bulk magnetization of the alloy is not considered in terms of magnetic and nonmagnetic atoms, but rather in terms of the average magnetic moment per each atom present. Let $N \uparrow$ and $N \downarrow$ be the number of valence electrons of each spin state. The chemical valence is

$$
Z=N \uparrow+N \downarrow
$$

and the magnetic moment per atom is

$$
\begin{aligned}
M & =N \uparrow-N \downarrow \\
& =2 N \uparrow-Z .
\end{aligned}
$$

In transition metals, the $N \uparrow$ electrons can be separated into the strongly delocalized $s, p$ electrons and the more localized $d$ electrons. Following Williams et al. [3], the concept of magnetic valence, $Z_{\mathrm{m}}$, may be introduced

$$
Z_{\mathrm{m}}=2 N_{d}^{\uparrow}-Z
$$

is an integer, and $M$ is deduced to be

$$
M=Z_{\mathrm{m}}+2 N_{s p}^{\uparrow} \text {. }
$$

The basic idea of this approach is that by replacing an atom of $\mathrm{Fe}$, Co or $\mathrm{Ni}$ by an $\mathrm{R}$ atom one removed precisely five $d$ states from below the Fermi level $E_{\mathrm{F}}$. In strong ferromagnets, the magnetic moment can thus be evaluated using Eq. (6) provided $Z_{\mathrm{m}}$ is replaced by

$$
\bar{Z}_{\mathrm{m}}=2 N_{d}^{\dagger}(1-y)-Z_{\mathrm{M}}(1-y)-y Z_{\mathrm{R}}
$$

where $Z_{\mathrm{M}}$ and $Z_{\mathrm{R}}$ refer to the chemical valence of the element $\mathrm{M}$ and $\mathrm{R}$ respectively. Assuming $2 N_{s p}^{\uparrow}=0.9$ [5], the calculated variation of the magnetic moment $M$ as a function of $\bar{Z}_{\mathrm{m}}$ corresponds to straight line.

In $\mathrm{Fe}$ compounds, the magnetic moments are below the calculated line, this corresponds to weak ferromagnetism. On the contrary, strong ferromagnetism is deduced to occur in Co-rich compounds. However, the magnetic moment per atom in the $\mathrm{Y}_{2} \mathrm{Co}_{7}$ compound and at larger $\mathrm{Y}$ concentration is lower than the calculated 
value. This means that strong ferromagnetism is at this point no longer stable. Actually, the splitting of the $3 d$ band vanishes entirely in $\mathrm{YCo}_{2}$ which is a Pauli paramagnet $[1,2]$.

\section{2. $3 d$ exchange interactions in $R-F e$ and $R$-Co compounds}

The Curie temperature of Co-based Y-Co alloys is close to that of pure Co at low $\mathrm{Y}$ concentrations, it decreases as the percentage of $\mathrm{Y}$ atoms is increased. In Y-Fe alloys, the Curie temperature is always significantly lower than that of pure $\mathrm{Fe}$, it increases as the percentage of $\mathrm{Y}$ atoms is increased. These variations in $T_{\mathrm{C}}$ can be due either to variations in the values of the local moments $\mu_{\mathrm{M}}$ or to variations in the strength of interactions between moments, represented by the molecular field coefficient $n_{\mathrm{MM}}$. In Co-based alloys, the Curie temperature is approximately proportional to $\mu_{\text {Co }}^{2}$. This shows that the interactions between local moments are not significantly affected by the changes in local environments from one alloy to another [7]. In the case of Fe alloys, in order to separate out the respective contributions of $\mu_{\mathrm{Fe}}^{2}$ and $n_{\mathrm{FeFe}}$ in the value of the magnetic interactions, it is meaningful to determine the value of the parameter $T_{\mathrm{C}} / \mu_{\mathrm{Fe}}^{2}$ which is proportional to $n_{\mathrm{FeFe}}$. These values decrease with the percentage of rare-earth atoms [6]. Although the $\mathrm{Fe}$ magnetic moment is larger in $\mathrm{Y}_{2} \mathrm{Fe}_{17}$ than in $\mathrm{YFe}_{2}$, the strong reduction in the molecular field coefficient $n_{\mathrm{FeFe}}$ for Fe-rich compounds is responsible for the value of $T_{\mathrm{C}}$ being lower in $\mathrm{Y}_{2} \mathrm{Fe}_{17}(319 \mathrm{~K})$ than in $\mathrm{YFe}_{2}$ $(570 \mathrm{~K})$. These large variations in $n_{\mathrm{FeFe}}$ from one compound to another can be attributed to a dependence of $n_{\mathrm{FeFe}}$ either on interatomic distances or on local environment effects. It is unlikely that the variations in $\mathrm{Fe}-\mathrm{Fe}$ interatomic distances from $\mathrm{R}_{2} \mathrm{Fe}_{17}$ to $\mathrm{RFe}_{2}$ which are of about $4 \%$ may be responsible for the observed increase in $n_{\mathrm{FeFe}}$ by about a factor 3 . Thus, it must be concluded that the values of $n_{\mathrm{FeFe}}$ are strongly influenced by local environment effects. Such a behaviour has already been observed in pure $\mathrm{Fe}$, bcc $\alpha$-Fe being a ferromagnet which orders at $1043 \mathrm{~K}$ while fcc $\gamma-\mathrm{Fe}$ is not magnetically ordered. These phenomena may be described in terms of local coordination of $\mathrm{Fe}$ atoms, $c_{n}$. The interactions between Fe moments decrease regularly as $\bar{c}_{n}$ increases from $6\left(\mathrm{RFe}_{2}\right)$ to $10.3\left(\mathrm{R}_{2} \mathrm{Fe}_{17}\right)$. Moreover, ferromagnetism of pure $\alpha$-Fe $\left(\bar{c}_{n}-8\right)$ and the absence of ferromagnetism in $\gamma$ - $\mathrm{Fe}\left(\bar{c}_{n}-12\right)$ can be predicted. Band structure calculations permit the understanding of the above beliaviour [5]. Strong ferromagnetic interactions are associated with open structures in which the local coordination of $\mathrm{Fe}$ atoms is low. The interactions are strongly reduced and antiferromagnetism may even occur in the case of close packed structure, with high local coordination for the Fe atoms ( $\gamma-\mathrm{Fe}, \mathrm{R}_{2} \mathrm{Fe}_{17}$ compounds).

\subsection{Exchange interactions at the $R$ sites}

The spontaneous magnetization at $4.2 \mathrm{~K}$ in alloys of the $R \mathrm{RO}_{5}$ and $\mathrm{R}_{2} \mathrm{Fe}_{14} \mathrm{~B}$ series is well known. Across a given series, the magnetization is a maximum for the $\mathrm{Pr}$ and Nd compounds, and it is a minimum for Dy compounds. In fact, ferromagnetic coupling occurs in compounds with light $R$ elements, whereas ferrimagnetic coupling occurs in compounds with heavy $R$ elements. This means that across the 
whole $\mathrm{R}$ series, an antiparallel coupling between the Fe and $\mathrm{R}$ spin moments takes place. An interpretation of this phenomenon has been proposed by Campbell [8]. Due to the localized character of the $4 f$ electrons, no direct interaction occurs between the $4 f$ and $3 d$ moments. It is natural to assume that the interactions are mediated by the $5 d$ electrons. At the $\mathrm{R}$ sites, positive interactions occur between the $4 f$ and the $5 d$ electrons. The interactions between $3 d$ and $5 d$ electrons may be discussed considering that in $\mathrm{Fe}$, Co and $\mathrm{Ni}$ the $d$ band is more than half-filled whereas in $\mathbf{R}$ atoms it is less than half-filled. In such instance, antiferromagnetic coupling of spins is expected [9]. The combination of $4 f-5 d$ and $5 d-3 d$ interactions thus leads to an antiferromagnetic coupling of spins as observed.

The energy associated with the above coupling may be expressed as

$$
E_{\mathrm{RFe}}=-n_{\mathrm{RFe}} M_{\mathrm{R}}^{\mathrm{s}} M_{\mathrm{Fe}}^{\mathrm{s}},
$$

where $n_{\mathrm{RFe}}$ is a molecular field coefficient and $M_{\mathrm{R}}^{\mathrm{s}}$ and $M_{\mathrm{Fe}}^{\mathrm{s}}$ are the spin momonts of $R$ and Fe atoms respectively [10]. This energy term is responsible for the Curie temperature, $T_{\mathrm{C}}$, in compounds with magnetic $\mathrm{R}$ elements being substantially larger than that in compounds with nonmagnetic $R$ elements. For the $\mathrm{R}_{2} \mathrm{Fe}_{14} \mathrm{~B}$ compounds. $T_{\mathrm{C}}$ is a maximum in the $\mathrm{Gd}$ compound which corresponds to $M_{\mathrm{R}}^{\mathrm{s}}=7 \mu_{\mathrm{B}}$ being maximum for $\mathrm{Gd}$.

Neglecting $\mathrm{R}-\mathrm{R}$ interactions, the Curie temperature may. be expressed as

$$
T_{\mathrm{C}}=\frac{1}{2}\left(T_{\mathrm{Fe}}+\sqrt{T_{\mathrm{Fe}}^{2}+4 T_{\mathrm{RFe}}^{2}}\right),
$$

where $T_{\mathrm{Fe}}$ is the Curie temperature in the absence of $\mathrm{R}-\mathrm{Fe}$ exchange interactions, i.e. approximately the Curie temperature in compounds with nonmagnetic $R$ elements. $T_{\mathrm{RFe}}$ represents the strength of $\mathrm{R}-\mathrm{Fe}$ interactions

$$
T_{\mathrm{RFe}}=2\left(\left|g_{J}-1\right| / g_{J}\right) n_{\mathrm{RFe}} \sqrt{C_{\mathrm{R}} C_{\mathrm{Fe}}},
$$

where $C_{\mathrm{R}}$ and $C_{\mathrm{Fe}}$ are the Curie constants of $\mathrm{R}$ and $\mathrm{Fe}$ atoms respectively. The value of $n_{\mathrm{RFe}}$ was deduced from Eqs. (9) and (10) for the $\mathrm{R}_{2} \mathrm{Fe}_{14} \mathrm{~B}$ compounds. $n_{\mathrm{RFe}}$ decreases by about a factor of 3 from the Pr compound to the Tm compound. A similar behaviour has been observed in all $\mathrm{R}-\mathrm{M}$ compounds studied [10].

Considering the similarity in band structures across a given series of compounds, it is likely that the $5 d-3 d$ interactions are approximately constant. Thus, the variation in $n_{\mathrm{RFe}}$ must be attributed to a large decrease in the $4 f-5 d$ on-site interactions. Indeed, the distance between the $4 f$ and $5 d$ shells increases across the rare-earth series and its variation remarkably reflects that of $n_{\mathrm{RFe}}$.

\subsection{If magnetocrystalline anisotropy in $R-M$ compounds}

Large anisotropy may occur when a magnetized rare-earth atom is submitted to the CEF in the environment. In rare-earth metals this occurs only at low temperature. In $\mathrm{R}-\mathrm{M}$ compounds, the exchange interactions at the $\mathrm{R}$ sites essentially arise from transition metal atoms. They are still fairly high at room temperalure in compounds whose Curie temperature is much higher than $300 \mathrm{~K}$. Owing to this phenomenon, a large fraction of the anisotropy is preserved at room temperature.

The sign and strength of the anisotropy depend on the peculiar crystal structure considered. As a general rule, larger anisotropy may be obtained in compounds 
crystallizing in a structure of uniaxial symmetry $\left(\mathrm{RCo}_{5}\right)$ than in a structure of cubic symmetry $\left(\mathrm{RM}_{2}\right)$. Let us consider, for instance, the hexagonal $\mathrm{RCo}_{5}$ compounds. Each $R$ atom is surrounded by $2 R$ atoms along the sixfold $c$-axis. The negative $4 f$ electron density is attracted by the positive charges of the surrounding $R$ atoms, and tends to be elongated along the $c$-axis. Uniaxial anisotropy is thus obtained for rare-earth atoms where $\alpha_{J}$ is positive (prolate). In $\mathrm{SmCo}_{5}$ the anisotropy energy at room temperature is $2 \times 10^{7} \mathrm{~J} / \mathrm{m}^{3}$ [11]. In the $\mathrm{R}_{2} \mathrm{Fe}_{14} \mathrm{~B}$ compounds the surrounding of the $R$ atoms is such that the $4 f$ electron density tends to be confined in plane perpendicular to $c$. There, uniaxial anisotropy occurs in the case of oblate $4 f$ electron density. In $\mathrm{Nd}_{2} \mathrm{Fe}_{14} \mathrm{~B}$, the anisotropy energy at room temperature is $5 \times 10^{6} \mathrm{~J} / \mathrm{m}^{3}[12]$.

\section{Magnetic thin films}

During the last few years important technical advances have been reached in the fabrication of artificial materials such as thin films, sandwiches, multilayers. Such materials can be prepared by sputtering with an ion gun under good vacuum or by evaporation using effusion cells or electron guns. With sophisticated techniques such as molecular-beam epitaxy, the growth of films can almost be controlled layer by layer.

Due to the short-range nature of magnetic interactions (exchange, CEF) the basic magnetic propertics must be studied in ultra-thin layers or very close to the surface. Areas of interest are in particular: (i) change of magnetic properties (moment, ordering temperatures, anisotropy) with thickness; (ii) preparation of artificial materials using epitaxial growth $(\gamma-\mathrm{Fe}, \mathrm{bcc}-\mathrm{Co})$. Some significant results obtained up to now are summarized in this section.

At intermediate thicknesses (few thousands $\AA$ ), the interest is focused on the preparation of new materials suitable for technological applications, in particular magnetic recording. Interesting properties of these materials are described in Sec. 5.

\subsection{Magnetic moments at surfaces and interfaces}

In a magnetic material, the environment of atoms at the surface differs from that of atoms in the bulk. Due to the lower coordination of surface atoms, the exchange interactions are reduced and it was originally thought that the moment at the surface may be zero ("magnetically dead layer") [15]. An evaluation of the value of the $\mathrm{Fe}$ moment at the surface of iron or at the interface in $\mathrm{Fe}-\mathrm{M}^{\prime}$ multilayers $\left(\mathbf{M}^{\prime}=\right.$ nonmagnetic atoms) can be deduced from measurement of the $\mathrm{Fe}$ hyperfine field, which is approximately proportional to the $3 d$ magnetic moment. This hyperfine field, measured in $\mathrm{Fe}$ metal [16] and at the interface in $\mathrm{Fe}-\mathrm{Ag}$ multilayers [17] is given in Table layer by layer. The hyperfine field at the surface does not differ significantly from the one in the bulk which precludes the existence of a dead layer. Similar results were also derived from the studies of several $\mathrm{Fe}-\mathrm{M}^{\prime}$ multilayers using polarised neutron diffraction [18].

Theoretical calculations of the Fe magnetic moment [19] are also given in Table. The moment at the surface is enhanced by about $20 \%$ from the bulk value. 
TABLE

Experimental Fe hyperfine field layer-by-layer in $\mathrm{Fe}[16]$ and at $\mathrm{Fe}-\mathrm{Ag}$ interface [17]. Calculated Fe magnetic moments [19].

\begin{tabular}{|c|c|c|c|}
\hline & \multicolumn{2}{|c|}{$\begin{array}{c}\text { Hyperfine field } \\
{[\mathrm{T}]}\end{array}$} & \multirow{2}{*}{$\begin{array}{c}\text { Calculated } \\
\text { Fe moment } \\
{\left[\mu_{\mathrm{B}}\right]}\end{array}$} \\
\hline & $\mathrm{Fe}$ & $\mathrm{Fe}-\mathrm{Ag}$ & \\
\hline$S$ & -33.2 & -34.8 & 2.65 \\
\hline$S-1$ & -34.7 & -34.5 & 2.37 \\
\hline$S-2$ & - & -34.4 & 2.28 \\
\hline$S-\mathbf{3}$ & - & -34.2 & 2.25 \\
\hline $\begin{array}{l}S-4 \\
\text { (bulk) }\end{array}$ & -34.0 & -34.0 & 2.22 \\
\hline
\end{tabular}

As explained by Freeman et al. [19], the surface atoms have lower coordination and reduced symmetry. This leads to a stronger localization of $3 d$ electrons around surface atoms and hence to an enhancement of the magnetic moment. Similar behaviour is predicted to occur for Co and $\mathrm{Ni}$. A very interesting case is $\mathrm{Cr}$ which in the bulk is an antiferromagnet with a moment of about $0.5 \mu_{\mathrm{B}}$. Angle resolved photoemission experiments indicate that ferromagnetism occurs at the surface [20], with a magnetic moment of about $2 \mu_{\mathrm{B}}$. This is in agreement with calculations which predict a surface magnetic moment of $2.49 \mu_{\mathrm{B}}$ [19].

\subsection{Ordering temperatures in thin films}

The Curie temperatures of $\mathrm{Fe}, \mathrm{Co}$, Ni thin films sandwiched between layers of $\mathrm{Au}$ have been deduced, in particular, from magnetization measurements with a SQUID magnetometer [21] or using magneto-optic Kerr effect [22]. The Curie temperature in nickel is not significantly reduced with respect to the bulk in films thicker than about $15 \AA$, but it decreases rapidly below this value. No magnetic ordering is observed in $\mathrm{Ni}$ monolayer. In $\mathrm{Fe}$ and $\mathrm{Co}$, the dependence of $T_{\mathrm{C}}$, which is higher than $1000 \mathrm{~K}$ for bulk specimen, cannot be easily determined. Nevertheless, it appears that ferromagnetism above room temperature is still present in monolayers.

The decrease in the Curie temperature in $\mathrm{Ni}$ at thicknesses below about $15 \AA$ can be related to the observation, in the paramagnetic state of bulk specimen, of strong ferromagnetic correlations [23]. This was ascribed to the itinerant nature of the $3 d$ magnetic electrons. In the paramagnetic state, the time $\ell_{\mathrm{h}}$, which characterizes hoping of electrons from site to site, is much shorter than the time, $l_{\mathrm{th}}$, which characterizes thermal fluctuations of the atomic moments. Thus, short range order occurs in the volume span by $3 d$ electrons in a time of the order of $t_{\mathrm{th}}$. 


\subsection{Magnetic anisolropy}

Large $3 d$ magnetic anisotropies have been observed at the surface of metals [24] or on monolayers [25]. In particular, Jonker et al. [25] deduced from spin-polarised photoemission that the easy direction of magnetization is perpendicular to the plane of an $\mathrm{Fe}$ monolayer.

In bulk $3 d$ transition metals, the spin-orbit coupling is weaker than CEF interactions. Then, in cubic symmetry, the orbitals are such that the orbital moment is weak. This leads to a small anisotropy in these metals. However, in uniaxial symmetry, the orbitals favoured by CEF interactions may assume a large orbital moment. In the hexagonal compound $\mathrm{YC}_{5}$, a room temperature Co anisotropy of $5.8 \times 10^{6} \mathrm{~J} / \mathrm{m}^{3}$ is observed. Similarly, the presence of a large surface anisotropy in Fe must be ascribed to the reduced symmetry for surface atoms. Band structure calculations [25] predict in the case of $\mathrm{Fe}$ a value of $3.5 \times 10^{6} \mathrm{~J} / \mathrm{m}^{3}$. This is larger than the shape anisotropy due to dipolar interactions $\left(\approx 2.5 \times 10^{6} \mathrm{~J} / \mathrm{m}^{3}\right)$ and thus would impose the direction of the moments to be perpendicular to the surface as observed experimentally.

\section{Sandwich thin films}

We have recently studied the magnetic properties of sandwich films of the type $\mathrm{R}-\mathrm{Co} / \mathrm{R}^{\prime}-\mathrm{Co} / \mathrm{R}-\mathrm{Co}$ in which $\mathrm{R}$ is a magnetic rare-earth element and $\mathrm{R}^{\prime}$ is nonmagnetic $Y$. In these systems, the exchange interactions are homogeneous throughout the whole film thickness but the magnetization direction alternates from one layer to another. A transition is observed under a field $H_{\mathrm{t}}$, which corresponds to the reversal of the magnetization which is initially antiviarallel to the field. The transition is associated with the formation of a Bloch wall in the Co sublattice, which allows the exchange interactions at the interface between two layers to be minimized. The presented results constitute an extension of this work $[26,27]$.

\subsection{Experimental}

A sandwich film was prepared by DC triode sputtering method. It consisted of a soft amorphous Co-Zr layer $4000 \AA$ thick sandwiched between two types of $2000 \AA$ thick Sm-Co multilayers with different coercivities. The hysteresis cycle and the susceptibility of this system were measured. The magnetization reversal mechanisms in such systems where a Bloch wall is created at the interface between two layers were also studied in a $\mathrm{Y}-\mathrm{Co} / \mathrm{Gd}-\mathrm{Co}$ bilayer by means of the transverse Kerr effect.

\subsection{Results and discussion}

The magnetic behaviour at $4.2 \mathrm{~K}$ of a $\mathrm{SmCo} / \mathrm{CoZr} /(\mathrm{SmCo})^{\prime}$ sandwich is shown in Fig. 1. The coercive field characterizing magnetization reversal in the $\mathrm{Co}-\mathrm{Zr}$ central layer is $25 \mathrm{Oe}$. It is larger than in a $\mathrm{Co}-\mathrm{Zr}$ single layer $(\approx 4 \mathrm{Oe})$ due to the energy lost in domain wall formation at each interface. A transition 


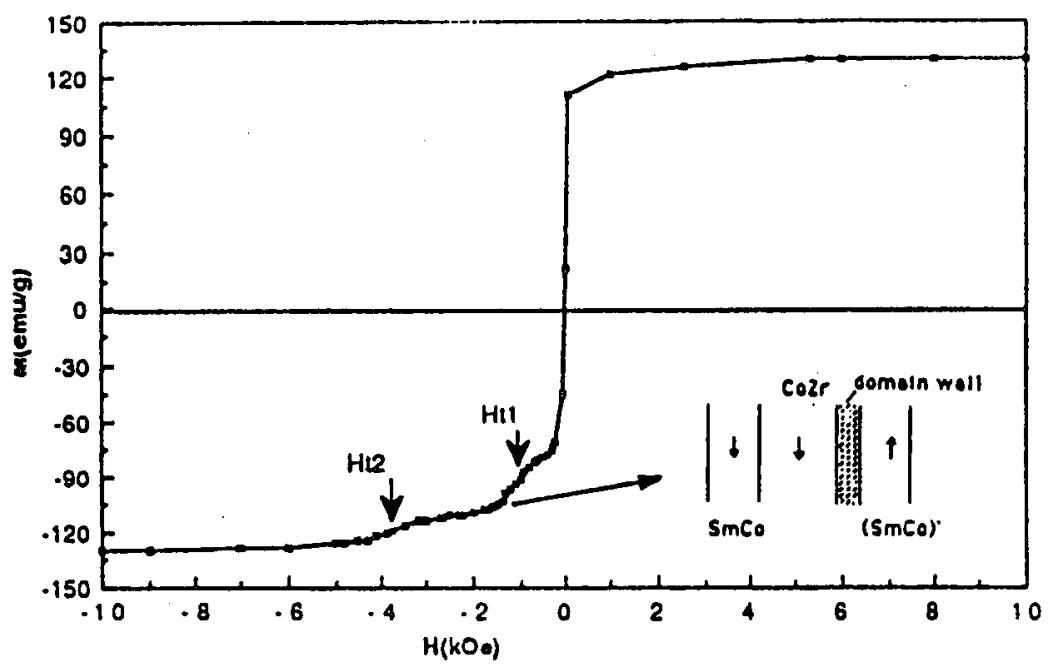

Fig. 1. Field dependence of the magnetization in a $\mathrm{SmCo} / \mathrm{CoZr} /(\mathrm{SmCo})^{\prime}$ sandwich film.

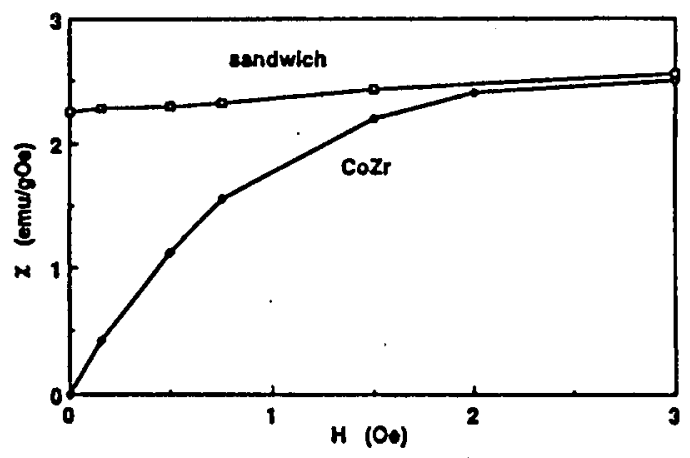

Fig. 2. Susceptibility in a $\mathrm{SmCo} / \mathrm{CoZr} /(\mathrm{SmCo})^{\prime}$ sandwich film and in a single $\mathrm{Co}-\mathrm{Zr}$ layer.

is observed at $H=H_{\mathrm{t} 1}=1.3 \mathrm{kOe}$. It corresponds to magnetization reversal in the less coercive Sm-Co layer. A second transition occurring at $H=H_{\mathrm{t} 2}=$ $4.1 \mathrm{kOe}$ results from magnetization reversal of the more coercive $\mathrm{Sm}-\mathrm{Co}$ laycr. The values of $H_{\mathrm{t} 1}$ and $H_{\mathrm{t} 2}$ are about half the values of $H_{\mathrm{c}}$ in each corresponding $\mathrm{Sm}$-Co single layer. This behaviour may be attributed to the different mechanisms involved depending whether magnetization reversal in the Sm-Co layer occurs by nucleation and growth from the saturated state (in the single layer) or by domain wall unpinning (in the sandwich).

A magnetization state of particular interest is stabilized by applying a field $H_{\mathrm{t} 1}<H<H_{\mathrm{t} 2}$ and then decreasing it to zero. In this state, the magnetization 


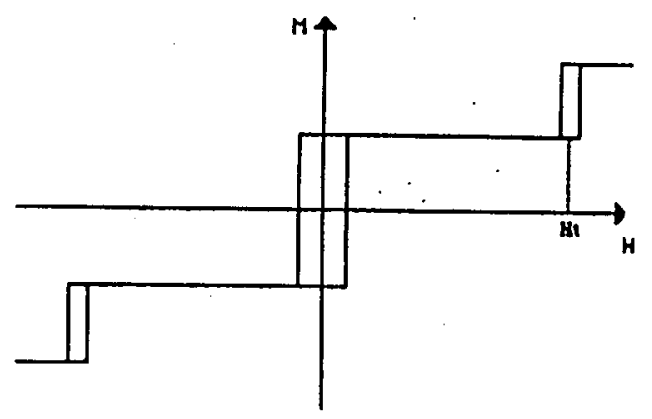

Fig. 3. Schematic representation of the hysteresis cycle in a $\mathrm{YCo} / \mathrm{GdCo}$ bilayer at $T=300 \mathrm{~K}$.

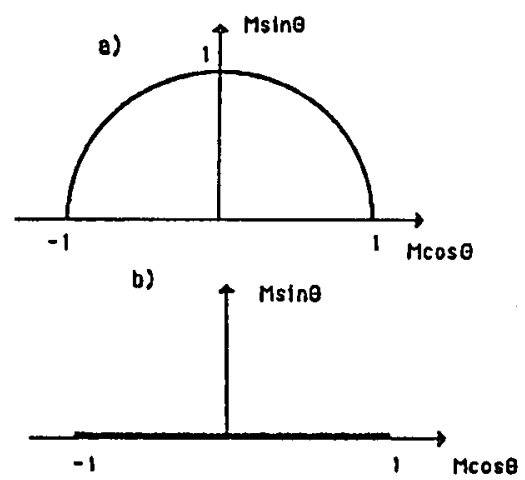

Fig. 4. $M \cos \theta$ versus $M \sin \theta$ for: (a) coherent rotation and (b) nucleation/propagation processes.

in bollh Sm-Co layers are of opposite directions and the central Co-Zr layer is divided by the domain wall into two parts. The susceptibility in this particular magnetization state was measured and compared to that of a single Co- $\mathrm{Zr}$ layer (Fig. 2). The low field susceptibility in the sandwich is 5 times higher than in the single layer and the magnetization processes are almost completely reversible. This properly can be explained by the fact that the domain wall formed along the interface in the Co-Zr layer has a very large area and therefore, unlike the case of usual domain walls, it almost does not interact with the structural defects in the film.

In order to study magnetization reversal mechanisms in this type of systems, a bilayer consisting of two amorphous YCo and GdCo layers was realized by DC triode sputtering. The thickness of the individual layers was $1000 \AA$. A schematic representation of the hysteresis cycle characterizing the bilayer is shown in Fig. 3 . At room tcmperature, a reversal of the magnetization as a whole occurs at the coercive field $H_{\mathrm{c}}=7 \mathrm{Oe}$. The transition observed at $H_{\mathrm{t}}=75 \mathrm{Oe}$ corresponds 
to the reversal of the magnetization in the GdCo layer whose magnetization is initially antiparallel to $H$ (the hysteresis of this transition is $3 \mathrm{Oe}$ ).

The longitudinal $(\| H)$ and transverse $(\perp H)$ components of the magnetization during reversal were determined simultaneously by transverse Kerr effect measurements. Schematically, if coherent rotation occurs, the magnetization vector describes a semi-circle in a $M \cos \theta$ versus $M \sin \theta$ plot (Fig. 4a). Alternatively, if nucleation/propagation occurs, the domains with $\theta=0$ grow at the expense of those with $\theta=\pi$, i.e. the transverse component remains 0 during the whole reversal process (Fig. $4 \mathrm{~b}$ ).

Magnetization reversal was observed on both YCo and GdCo sides of the bilayer. At $H=H_{\mathrm{c}}$, the behaviour observed is typical of a nucleation/propagation process. This could be expected as it is well known that coherent rotation, which would occur at $H=H_{\mathrm{A}}$, is always by-passed by nucleation at defects in lower fields [28].

The transition of the GdCo layer was observed at $H=H_{\mathrm{t}}$. Considering that a domain wall is then formed at the interface, it seemed plausible that coherent rotation could occur. Actually, the observed signal was identical to the one observed in the vicinity of $H=H_{\mathrm{c}}$, which indicates that here also magnetization reversal occurs by nucleation/propagation process (see Fig. $4 \mathrm{~b}$ ).

\section{References}

[1] K.H.J. Buschow, Rep. Prog. Phys. 40, 1179 (1977).

[2] H.R. Kirchmayr, C.A. Poldy, Handbook on the Physics and Chemistry of Rare Earths, Eds. K.A. Gschneidner, L. Eyring, North-Holland, Amsterdam 1979, p. 55.

[3] A.R. Williams, V.L. Moruzzi, A.P. Malozemoff, K. Terakura, IEEE Trans. Magn. MAG-19, 1983 (1983).

[4] J. Friedel, Nuovo Cimento Suppl. 2, 287 (1958).

[5] A.R. Williams, V.L. Moruzzi, D.C. Gellat, S. Kübler Jr., J. Magn. Magn. Maler. 31-34, 88 (1983). .

[6] J.P. Gavigan, D. Givord, H.S. Li, J. Voiron, Physica B 149, 345 (1988).

[7] D. Givord, R. Lemaire, IEEE Trans. Magn. MAG-10, 109 (1974).

[8] I.A. Campbell, J. Phys. F, Metal Phys. 2, L47 (1972).

[9] J. Friedel, Nuovo Cimento Suppl. 2, 287 (1958).

[10] E. Belorizky, M.A. Fremy, J.P. Gavigan, D. Givord, H.S. Li, J. Appl. Phys. 61, 3971 (1987).

[11] J. Laforest, Thesis, Grenoble University, Grenoble 1981.

[12] J.M. Cadogan, J.P. Gavigan, D. Givord, II.S. Li, J. Phys. F, Metal Plys. 18, L195 (1988).

[13] G. Hoffer, K.A. Strnat, IEEE Trans. Magn. MAG-2, 487 (1966).

[14] M. Sagawa, S. Fujimura, M. Togawa, II. Yamamoto, Y. Matsuura, J. Appl. Phys. 55, 2083 (1984).

[15] L.N. Lieberman, D.R. Fredkin, H.B. Shore, Phys. Rev. 22, 539 (1969).

[16] J. Korecki, U. Gradmann, Phys. Rev. Lett. 55, 2491 (1985). 
[17] J. Tyson, A.H. Owens, J.C. Walker, G. Bayreuther, J. Appl. Phys. 52, 2487 (1981). [18] Y. Endoh, N. Hosoito, T. Shinjo, J. Magn. Magn. Mater. 35, 93 (1983).

[19] S. Ohmishi, A.J. Freeman, M. Weinert, Phys. Rev. B 28, 6741 (1983).

[20] L.E. Klebanoff, S.W. Robey, G. Liu, D.A. Shirley, Phys. Rev. B 30, 1048 (1984).

[21] C. Chappert, D. Renard, P. Beauvillain, J.P. Renard, J. Phys. Lett. 46, L59 (1985).

[22] S.D. Bader, E.R. Moog, J. Appl. Phys. 61, 3729 (1987).

[23] P.J. Brown, H. Capellmann, J. Déportes, D. Givord, K.R.A. Ziebeck, J. Magn. Magn. Mater. 30, 243 (1982).

[24] U. Gradmann, J. Magn. Magn. Mater. 54-57, 733 (1986).

[25] B.T. Jonker, K.H. Walker, E. Kisker, G.A. Prinz, C. Carbone, Phys. Rev. Lett. 57, $142(1986)$.

[26] B. Dieny, D. Givord, J.M.B. Ndjaka, J.M. Alameda, J. Appl. Phys. 67, 5677 (1990).

[27] B. Dieny, D. Givord, J.M.B. Ndjaka, J. Magn. Magn. Mater. 93, 503 (1991).

[28] H. Zijlstra, in: Ferromagnetic Materials, Ed. E.P. Wohlfarth, North-Holland, Amsterdam 1982, p. 37. 